\title{
Cellular basis for paracrine regulation of ovarian follicle development*
}

\author{
David F. Albertini, Catherine M. H. Combelles, \\ Elizabeth Benecchi and Mary Jo Carabatsos
}

\author{
Department of Anatomy and Cellular Biology, Tufts University School of Medicine, Boston, \\ MA 02111, USA
}

\begin{abstract}
Paracrine factors secreted by oocytes and somatic cells regulate many important aspects of early ovarian follicle development in mammals. From activation of dormant primordial follicles to selection of secondary follicles, locally acting factors have been identified that appear to exert important effects on the growth and differentiation of oocytes and granulosa cells. This article summarizes evidence to support a model for bi-directional paracrine communication that is based on developmental regulation of the delivery and reception of paracrine factors at the oocyte-granulosa cell interface. Transzonal projections that originate from granulosa cells and terminate at the oocyte plasma membrane provide a polarized means to orient the secretory organelles of somatic cells. Characterization of transzonal projections in follicles from normal and genetically modified mice reveals dynamic changes in the density and stability of transzonal projections. On the basis of new data analysing the orientation and cytoskeletal content of transzonal projections in mammalian oocytes, a model is proposed for regulation of paracrine growth factor secretion by follicle-stimulating hormone. These findings have immediate implications for ovarian hyperstimulation protocols and follicle culture models as related to the production of mammalian embryos by assisted reproductive technologies.
\end{abstract}

\begin{abstract}
Although the physiological, molecular and genetic determinants that initiate and sustain follicular growth and maturation have received much attention (see reviews by McNatty et al., 1999; McGee and Hsueh, 2000), comparatively little information has been forthcoming with regard to the changes in cellular behaviour that attend the process of folliculogenesis. When viewed in the context of other developmental systems, in which paracrine control of cell differentiation is considered implicitly relative to cell signalling, cell-cell adhesion and cell-extracellular matrix (ECM) interactions, our knowledge of cellular behaviours in the ovarian follicle is lacking, despite the wealth of information available on the role of growth factors and hormones in ovarian follicle development (for a review see Elvin and Matzuk, 1998). Recently, Rodgers et al. (1999, 2000) have drawn attention to the role of the ECM during bovine follicle growth as related to changes in the follicular basal lamina that may influence the growth and differentiation of both granulosa and theca cells. Given the
\end{abstract}

Email: david.albertini@tufts.edu

*This review is based on a symposium talk given at the meeting of the Society for the Study of Fertility held at the Edinburgh International Conference Centre from 31 July to 2 August 2000. general importance of the ECM in regulating diverse cell behaviours including migration, division, adhesion and death, this direction of study will add measurably to our understanding of the cellular forces that shape remodelling of the follicle during normal growth, differentiation and atresia.

In the context of paracrine factor access and presentation to target cells, it is helpful to consider the disposition of the $\mathrm{ECM}$ relative to the three basic types of cell present in preantral follicles. Although much of the growth of preantral follicles is characterized by gradual expansion of the follicular basal lamina as oocyte size and the number of granulosa cells increase, thecal cells also associate with the follicle and begin their course of differentiation. Given that growth factors such as growth differentiation factor 9 (GDF9) and kit ligand $(K L)$ have been implicated in the paracrine control of early follicle development (Yoshida et al., 1997; Elvin and Matzuk, 1998; Elvin et al., 1999a; Galloway et al., 2000), it is essential to consider the cellular origins and targets of signalling molecules in relation to the cell-ECM interfaces present at these early stages. The follicular basal lamina encapsulates granulosa cells and separates these cells from the surrounding theca cells, which are encased by basal lamina-like material (see Rodgers et al., 2000). The 
'personalized' ECM of the oocyte, the zona pellucida, also becomes highly organized and serves to limit contact between the oocyte and surrounding granulosa cells. If oocytes, granulosa and theca cells act as cellular sources or receivers of paracrine growth factors like GDF-9 or KL, there must be mechanisms for the bi-directional exchange of such factors through these intervening matrices. Given this problem of the spatial partitioning of discrete types of cell by the ECM, and the well-known role of the ECM as a selective filtration or barrier device, this article reviews what is known about the oocyte-granulosa cell interface with respect to cellular mechanisms that could facilitate and regulate bi-directional exchange of paracrine signals (Buccione et al., 1990).

\section{Transzonal projections at the oocyte-granulosa cell boundary}

Transzonal projections (TZPs), follicle cell extensions that traverse the zona pellucida and terminate on the oocyte cell surface, have been well characterized in many mammals by electron microscopy (Hertig and Adams, 1967; Anderson and Albertini, 1976; reviewed in Motta et al., 1994). The studies of Motta et al. (1994) on human ovarian follicles were the first to demonstrate that dynamic alterations in the number and form of TZPs occur at specific stages of follicle development and might serve to co-ordinate or modulate information exchange between the oocyte and follicle cells. This work showed that, in preantral follicles, TZPs were most numerous forming both adhesive and gap junctional contacts at the oolemma, and further demonstrated that during peak periods of oocyte growth, TZPs extended as deep invaginations that impinged upon the germinal vesicle. After further antral follicle development, TZPs retracted and maintained fewer terminal connections with the oocyte than in preantral follicles. During ovulation, active retraction of TZPs was noted (Motta et al., 1994).

These seminal findings merit reinterpretation given recent observations on the composition and structure of TZPs and assembly of the zona pellucida. The continued synthesis and secretion of zona pellucida proteins throughout ovulation indicates that remodelling of the zona pellucida is an ongoing process during folliculogenesis (Wassarman and Albertini, 1994). Since there is abundant evidence consistent with the contention that cumulus cell uncoupling from the oocyte involves TZP retraction and remodelling during maturation of cumulus-oocyte complexes in vivo and in vitro (Allworth and Albertini, 1993; Albertini and Rider, 1994; Suzuki et al., 2000), the continued synthesis and secretion of zona proteins may allow for closure of channels left after cellular retraction. Thus, marked changes in granulosa-oocyte and granulosa-granulosa cell adhesion, induced by the LH surge at ovulation, co-ordinate changes in the shape and secretory activity of cumulus cells with the final stages of oocyte and follicle maturation. But what about earlier transitions in follicle development?

Differences in gonadotropin-dependent events in antral follicle development, during what McGee and Hsueh (2000) refer to as cyclic recruitment, probably involve very different signalling requirements compared with the initial recruitment of primordial follicles into the growing pool of preantral follicles. Plasticity and remodelling of the zona pellucida may similarly allow for regulated changes in the establishment and maintenance of functional TZPs during these different critical transitions of follicle development.

The observations of Motta et al. (1994) on preantral follicles should be considered in light of the predominant, but not exclusive, role of known paracrine factors during these early developmental stages. TZPs were reported to be most abundant in preantral follicles. Studies in our laboratory have defined functional properties of TZPs in preantral follicles that would support their role in the localized secretion or uptake of factors at the oocytegranulosa cell interface (Can et al., 1997). Numerous microtubule-containing TZPs are present within the zona pellucida in preantral follicles as evidenced by optical sectioning of intact follicles by confocal microscopy (Fig. 1a). Moreover, mitochondrial vital staining of intact cultured mouse follicles with MitoTracker ${ }^{\mathrm{TM}}$ shows active bi-directional movements of these organelles within TZPs when monitored by time-lapse confocal microscopy (Fig. 1b). Similarly, loading follicles with non-metabolizable and neutral charge endocytic tracers like fluorescent dextrans shows active translocations of endosomes and lysosomes across the zona pellucida due to organelle movement within TZPs (Fig. 1c). These findings extend ultrastructural observations on the organellar contents of TZPs and document their capacity to support polarized organelle movement, an observation consistent with the demonstration of microtubules within TZPs and the role of microtubules in directed organelle movement (Allworth and Albertini, 1993; Can et al., 1997; Carabatsos et al., 1998). If these structures mediate directed transport, secretion, or selective uptake of factors secreted by the oocyte (for example GDF-9), it would be expected that granulosa cells apposed to the zona pellucida would be anchored and oriented to support localized macromolecular exchange. This contention is supported by electron microscopy of normal follicles and follicles from mutant lines of mice blocked at various stages of preantral development.

In control mice, TZPs observed in preantral follicles are anchored by F-actin bearing follicle cell extensions at the periphery of the zona pellucida, but send solitary and usually corkscrew-shaped projections through the zona pellucida (Fig. 2a). Follicles from GDF-9 null (-/-) animals lack anchoring TZPs but show prominent organelle-rich TZPs that envelope the oocyte surface, indicating that, in this situation, an unregulated exploration of the oocyte is allowed to occur (Carabatsos et al., 1998; Fig. 2b). In marked contrast, animals null for the $\mathrm{FSH}-\beta$ gene, in which follicle development is arrested at the late secondary preantral stage (Kumar et al., 1997), show prominent organelle-rich and anchoring TZPs (Fig. 2c). Furthermore, 

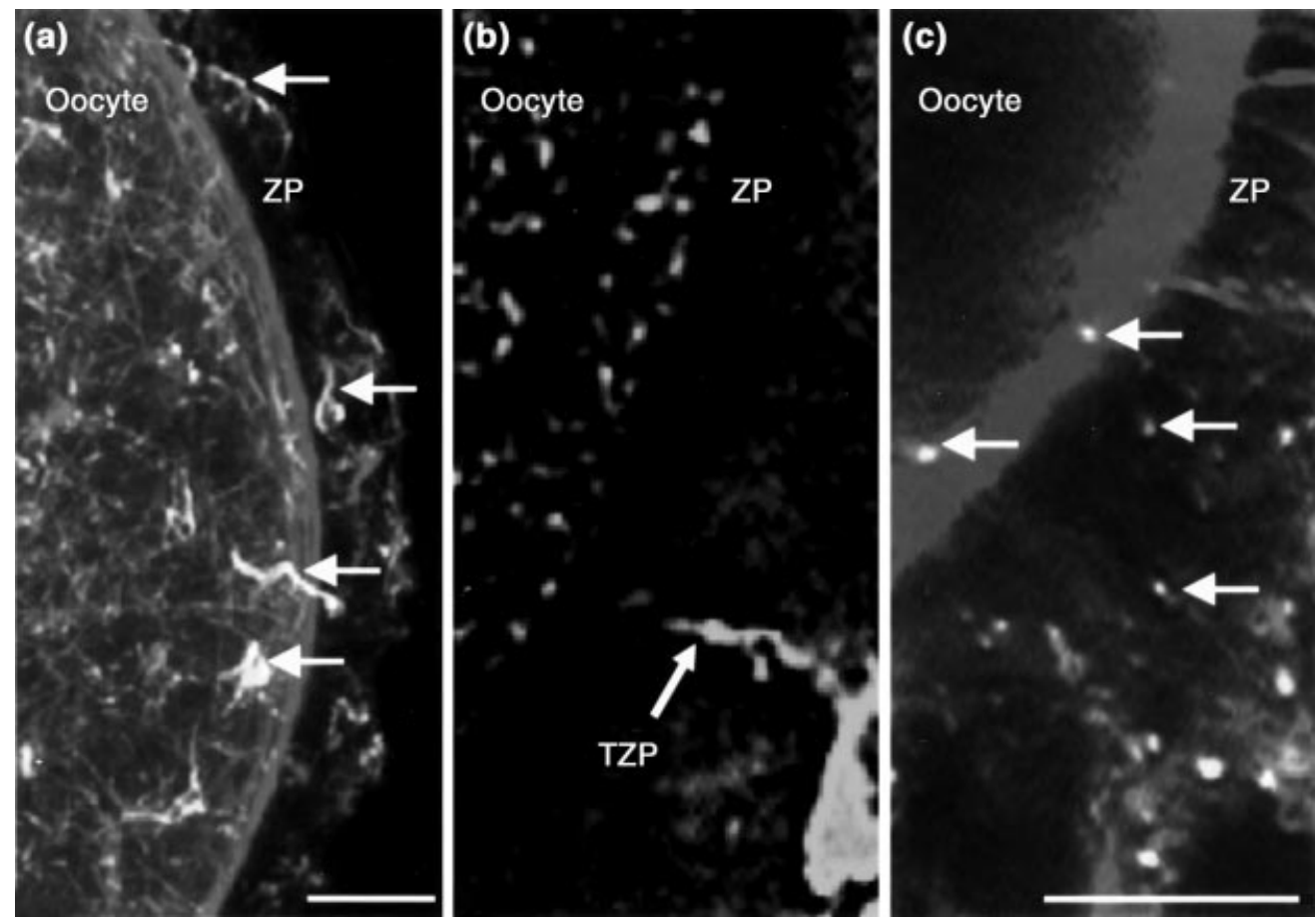

Fig. 1. Confocal microscope images of intact preantral mouse ovarian follicles. (a) Dense network of microtubule-containing transzonal projections (TZPs) derived from follicle cells (arrows). (b) A single confocal image of vitally stained mitochondria within a follicle cell TZP (arrow) obtained from a living cultured follicle labelled with MitoTracker ${ }^{\mathrm{TM}}$. (c) Fluorescent dextran-labelled endosomes (arrows) within follicle cell TZPs from a follicle cultured in the presence of endocytic marker before fixation and analysis by confocal microscopy. ZP: zona pellucida. Scale bars represent $5 \mu \mathrm{m}$.

the centrosome-Golgi complex is oriented towards the oocyte in nearly all follicle cells apposed to the outer surface of the zona pellucida in this FSH- $\beta$-deficient animal model (Fig. 2c). Thus, in the absence of FSH, follicle arrest at the secondary stage is associated with a striking degree of granulosa cell polarity that spatially approximates the secretory machinery with an increased density of TZPs. The implications of this finding are discussed below with respect to processing, secretion and transcytotic transport of factors between oocyte, granulosa and theca. In addition, a central question that emerges from observations of enhanced input in the absence of FSH- $\beta$ is: what regulates the orientation and stability of TZPs?

\section{Factors regulating TZP stability}

As noted earlier, changes in cell-cell (junctional) and cell-zona adhesion underscore both the retraction of TZPS and the migration of cumulus cells during hormone-induced expansion (Albertini and Rider, 1994), and, in some systems, selective loss of anchoring of granulosa cells to the zona pellucida is associated with invasion of microtubule TZPs during hormone-induced oocyte maturation (Allworth and Albertini, 1993). As in axons, microtubules form the cytoskeletal core of granulosa cell TZPs and would provide tracks for the polarized translocation of secretory pathway organelles. Thus, it is important to consider factors intrinsic to granulosa cells that influence the stability of TZPs, since, as indicated earlier, developmental changes in their stability would have direct consequences on the processing and action of paracrine factors originating from the oocyte or somatic follicular cells.

One mechanism for stabilizing microtubules involves post-translational acetylation of the $\alpha$-tubulin subunit that occurs subsequent to microtubule polymerization (Can and Albertini, 1997). Cultured human ovarian granulosa cells show a subpopulation of drug-resistant acetylated microtubules (Can and Albertini, 1997) and these are present in human cumulus cells. Typically, all TZPs present in the zona pellucida of human oocytes retrieved for in vitro fertilization show acetylated microtubules (Fig. 3). In addition to the staining observed within branching TZPs, staining is found within circular hoop-like structures that represent terminal portions of TZPs which associate with the oocyte surface (Fig. 3b, arrows). Thus, the prevalence of acetylated microtubules within TZPs of human oocytes indicates that this mechanism for maintaining microtubule stability may persist through the latest stages of follicle development to maintain oocyte-granulosa cell connections during cumulus expansion. This mechanism for TZP stabilization has been evaluated in follicles at earlier developmental stages. 

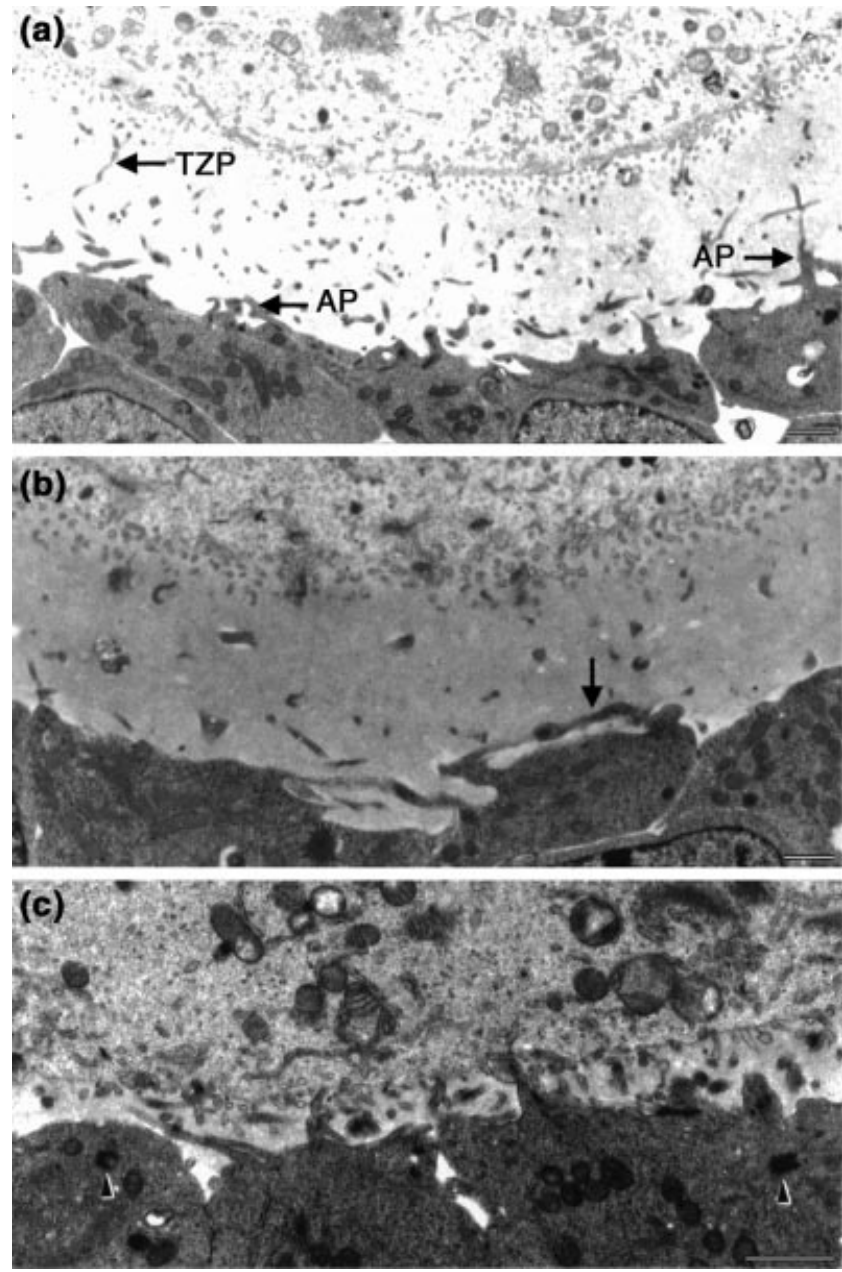

Fig. 2. Electron micrographs of the oocyte-granulosa cell interface from control (a), growth differentiation factor 9 (GDF-9) null (b) or FSH- $\beta$ null (c) mouse preantral follicles. In control follicles, anchoring projections (AP) and zona-traversing projections (TZP) are evident (a). Prominent organelle-rich TZPs (arrow) are typical in GDF-9 null follicles (b). Follicles from FSH- $\beta$ null animals show a striking degree of granulosa cell orientation towards the oocyte as evidenced by the apposition of the centrosome-centriole complex (c, arrowheads). Scale bars represent $1 \mu \mathrm{m}$.

A similar approach was used to analyse TZPs in preantral mouse follicles with respect to the possible consequences of protein kinase A activation in granulosa cells, one of the most important signalling pathways invoked to regulate granulosa cell differentiation (McGee and Hsueh, 2000). Untreated oocytes isolated from preantral follicles of prepubertal mice show a high density of TZPs that are enriched in the acetylation epitope (Fig. 4a,b). Brief treatments with the phosphodiesterase inhibitor isobutylmethylxanthine (IBMX), an agent commonly used to increase intracellular cyclic monophosphate concentrations in granulosa cells, causes both a reduction in TZP density and a decrease in immunodetectable acetylated tubulin (Fig. 4c,d). Together, these observations imply that
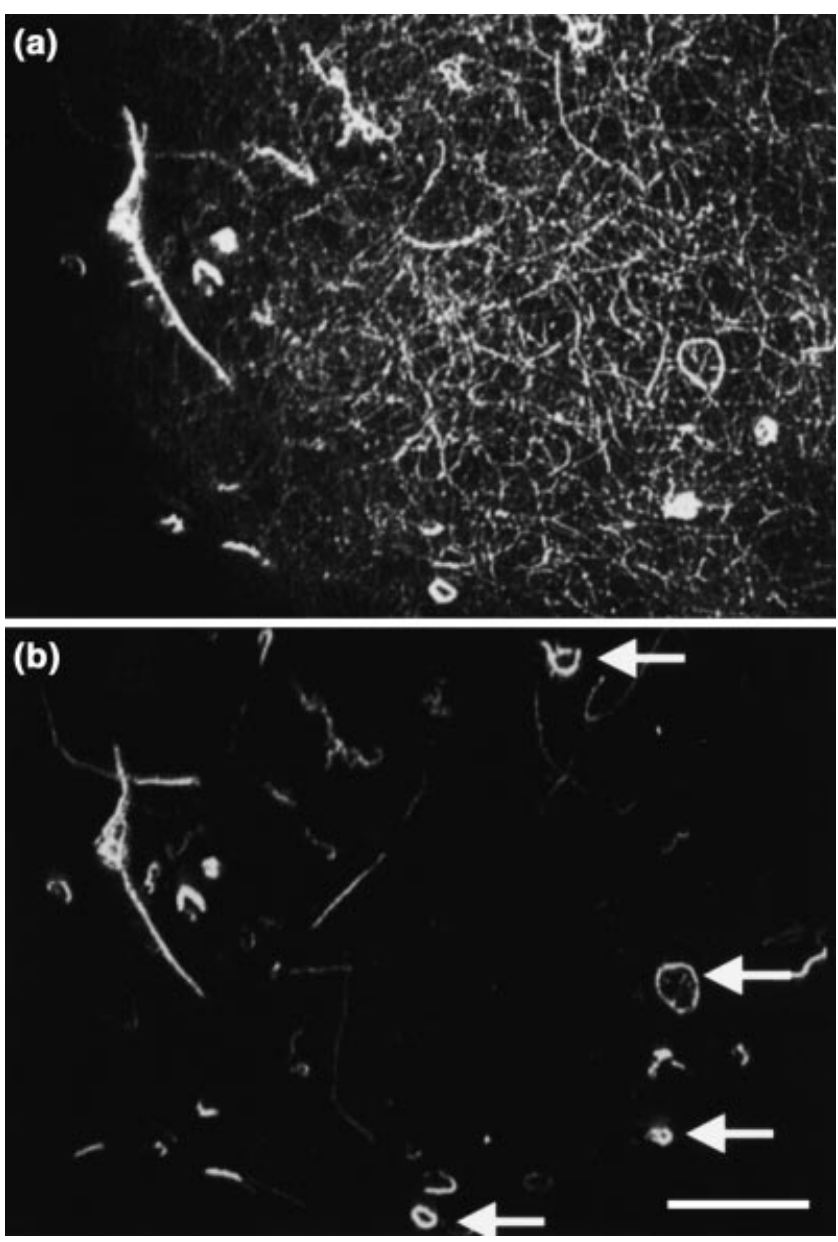

Fig. 3. Comparison of total tubulin (a) and acetylated tubulin (b) staining patterns within zona-enclosed human oocytes retrieved for in vitro fertilization. Note the abundance of oocyte microtubules (a) that lack acetylated $\alpha$-tubulin epitope after immunostaining compared with those within TZPs with acetylated $\alpha$-tubulin epitope (b). TZPs containing acetylated microtubules often terminate as hoops (arrows) that are located at the oocyte surface. Scale bar represents $20 \mu \mathrm{m}$.

TZPs are dynamic granulosa cell structures, the stability and function of which may be subject to both developmental and hormonal regulation. As specialized conduits for the transport, processing and reception of paracrine factors at the oocyte-granulosa cell interface, TZPs may represent a novel mechanism for the regulation of paracrine signalling in the developing follicle. A model for this hypothesis is presented below.

\section{Regulation of paracrine signalling}

Given the importance of modulating local forms of cell communication within the developing ovarian follicle, especially with respect to the changing demands for oocyte-granulosa cell cross talk at different developmental stages, this review of cellular behaviours has focused on 

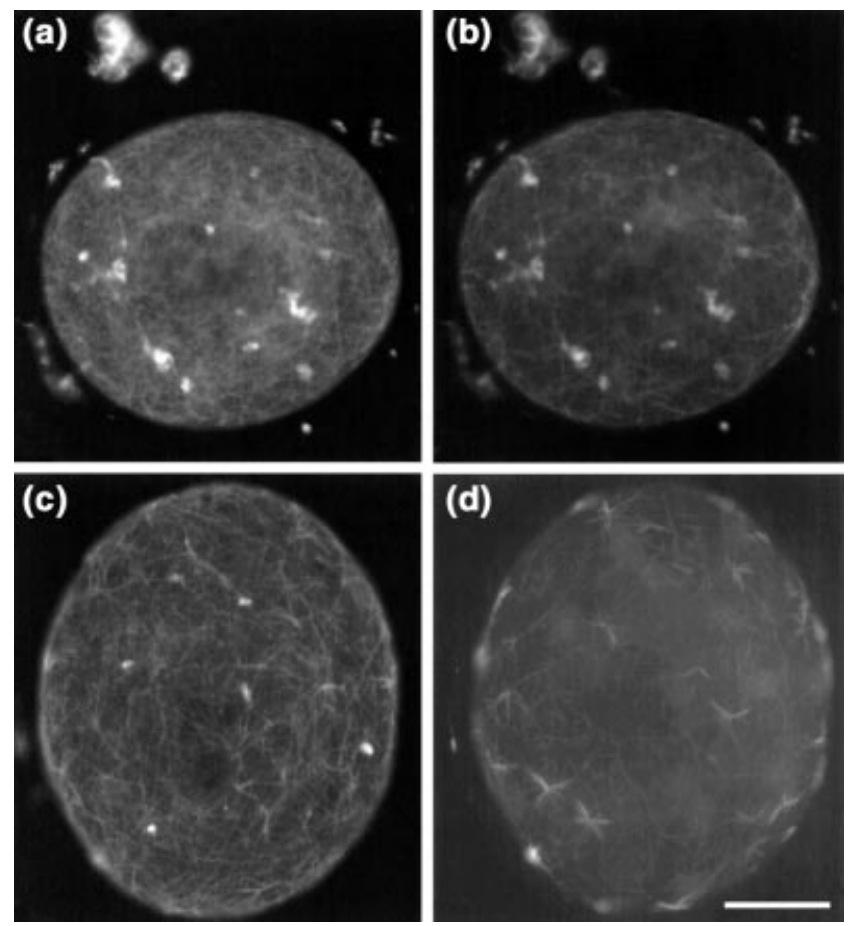

Fig. 4. Effects of isobutylmethylxanthine (IBMX) on the density and tubulin acetylation of TZPs in mouse oocytes isolated from preantral follicles. Total tubulin (a) and acetylated tubulin (b) in control oocytes cultured for $2 \mathrm{~h}$ before fixation and processing. (c,d) Respective tubulin patterns after exposure to $100 \mu \mathrm{mol}$ IBMX $\mathrm{I}^{-1}$ for $2 \mathrm{~h}$; note the reduction in TZP density and expression of acetylation epitope. Scale bar represents $20 \mu \mathrm{m}$.

two important points. First, modifications in cell-cell and cell-matrix interactions are likely to influence both the availability and actions of growth factors derived from each of the cellular compartments of the follicle (oocyte, granulosa and theca). Thus, the architectural remodelling associated with oocyte growth, epithelial expansion, antrum formation and differentiation of a vascularized theca represents significant consequences of differential gene expression that both reinforce functional distinctions in the germ and somatic cell lineages of the follicle and coordinate the processes of oogenesis and folliculogenesis. Second, since oocyte development is a critical aspect of follicle development and oocytes exert profound effects on granulosa cells, the interface between oocyte and granulosa cell emerges as a major control site for the co-ordination of follicle development. Historically, the properties of this interface have been viewed as fundamental to the regulation of oocyte growth and maturation and follicular luteinization (Elvin et al., 1999a,b, 2000; Mermillod et al., 1999; Nagyova et al., 2000). Here, we provide a novel framework for considering aspects of paracrine signalling that would depend directly on dynamic changes in the connections between oocyte and granulosa cells.

On the basis of work summarized earlier in this review, the model proposed (see Fig. 5) has been developed to explain how the interplay between growth factors and hormones may mediate the selection, support and demise (atresia) of the follicle. Implicit in this model is the notion that TZPs serve as specialized devices for mediating information exchange between oocyte and granulosa cells. This exchange includes, but is not limited to, the exchange of small molecules via gap junctions that support metabolite exchange. Thus, in preantral follicles, in which oocyte growth is supported and follicle growth is protracted, bidirectional exchange of cytoplasmic signals, whether nutrients or second messengers, co-ordinates directly certain aspects of metabolism shared by oocytes and granulosa cells (Buccione et al., 1990). Possibly just as important is the need to establish and maintain terminal TZP connections that would mediate both the local delivery of growth factors and possibly facilitate receptor occupation and activation by ligands like KL, a granulosa cell product (Yoshida et al., 1997; Elvin et al., 1999a). Delivery of follicle cell-derived leptin and STAT3 to the oocyte by TZPs has been reported (Antczak and Van Blerkom, 1997). TZPs would also support uptake of oocyte products, like oocyte-specific members of the transforming growth factor $\beta$ superfamily (GDF-9), which could be transported and possibly processed during transcytosis through granulosa cells for presentation to more distal granulosa cells and even surrounding theca (Dube et al., 1998; Solovyeva et al., 2000). Central to this model are key properties of TZPs: (i) that they possess the appropriate subcellular machinery for orienting the trafficking of paracrine factors; and (ii) that their dynamics are controlled by other factors (for example FSH) such that localized delivery and uptake is regulated. It appears that criteria to support the idea of polarized secretion and endocytosis have been met, at least on a preliminary level and, as the model proposes, FSH may be one factor causing TZP retraction given the data mentioned above.

In many ways, TZPs resemble axons, as has been previously alluded to (Allworth and Albertini, 1993), and recent data indicate additional similarities to synapse-like modes of signal transmission (Grosse et al., 2000). The cytoskeletal composition, organellar contents and overall disposition of TZPs at the oocyte-granulosa cell interface (Motta et al., 1994) support a view consistent with TZPs functioning in the directed delivery and uptake of factors within the perivitelline space. Although future work will be needed to define the factors involved in the formation and function of TZPs, especially with respect to the actions of GDF-9, KL and related signalling molecules, there is already some evidence to support their role in the acquisition of developmental competence by mammalian oocytes (Liu et al., 1997; Carabatsos et al., 1998; Mermillod et al., 1999). As mentioned, Antczak and Van Blerkom (1997) have shown that leptin and STAT3, factors that may influence embryo metabolism, assume a polarized distribution in oocytes and embryos. On the basis of confocal microscopy studies in intact follicles, it was proposed that leptin and STAT3 are maternally derived from a subpopulation of 


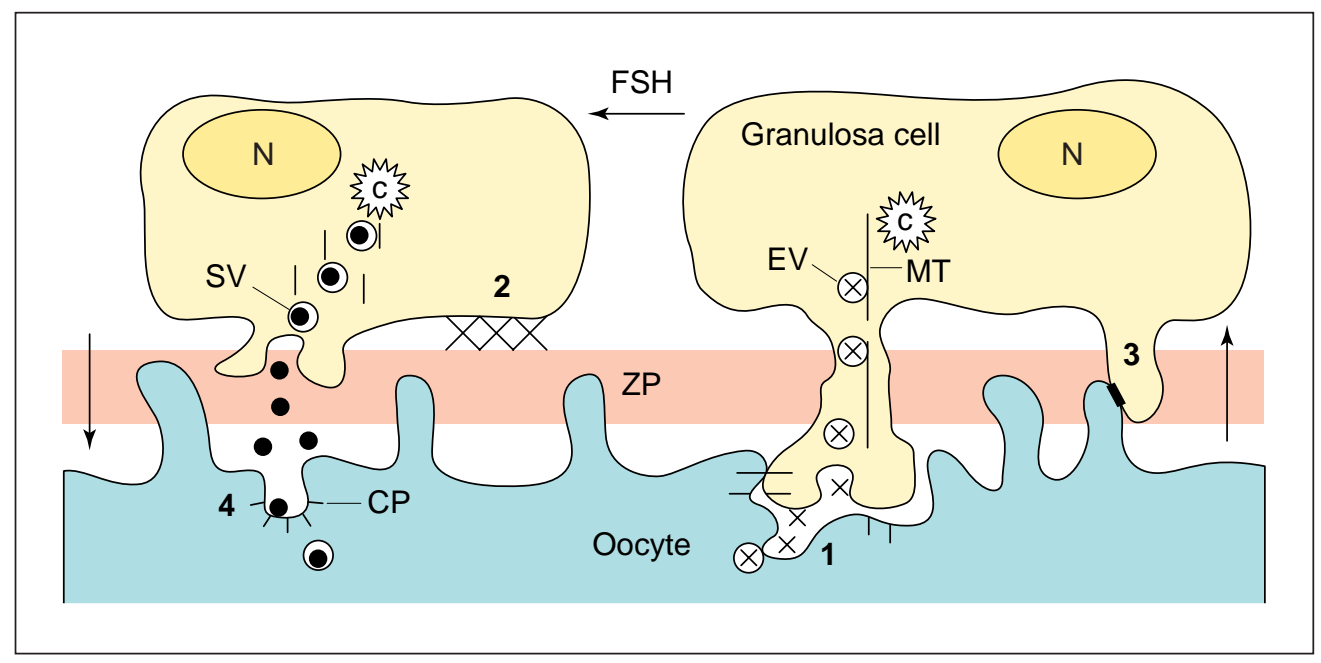

Fig. 5. Model proposing regulated delivery of paracrine factors at the oocyte-granulosa cell interface. Four (1-4) communication modalities are described. (1) Localized uptake of oocyte factors $(X)$, like GDF-9, by endocytosis at stabilized attachment sites of transzonal projections (TZPs) at the oolemma; vectorial transport of endocytic vesicles (EV) to granulosa cell body occurs along microtubules (MT) in preparation for intracellular processing and release of factors after transcytosis. (2) Granulosa-zona pellucida anchoring required for TZP orientation. Contact sites may play a signalling role for oocytes and granulosa cells, and changes in adhesion would occur in response to changes in composition of the zona pellucida. (3) Gap junctions that allow for direct intercellular communication between oocyte microvilli and granulosa cell TZPs. (4) Pathway for delivery of granulosa cell-derived factors packaged in secretory vesicles (SV) that are subsequently endocytosed by receptor-mediated endocytosis at the oocyte surface through coated pits (CP). Note that remodelling of the microtubule cytoskeleton (MT) in response to granulosa cell stimulation by FSH would lead to TZP retraction and thus modulating either the delivery of granulosa cell factors $(\bullet)$ to the oocyte (arrow, left) or uptake of oocyte secreted factors $(X)$ by granulosa cells (arrow, right). c: centrosome; FSH: follicle-stimulating hormone; N: nucleus; MT: microtubules; ZP: zona pellucida; $\bullet$ : granulosa cell factors; $\times$ : oocytesecreted factors.

follicle cells and that TZPs deliver these products into the perivitelline space for endocytosis by the oocyte.

The extent to which these and other protein factors secreted by granulosa cells gain access to the oocyte needs to be investigated more thoroughly given current ART protocols that involve removal of somatic cells. This commonly practised manipulation may compromise efficient delivery of factors to the oocyte at crucial stages of development that normally would have been subserved by the function of TZPs proposed here. In light of the data presented herein, the success of hormone-induced follicle development in vivo or in vitro may depend on experimental protocols that maintain the structural integrity of the granulosa-oocyte cell interface (Hayashi et al., 1999).

\section{References}

Key references are identified by asterisks.

Albertini DF and Rider V (1994) Patterns of intercellular connectivity in the mammalian cumulus-oocyte complex Microscopy Research and Technique 27 125-133

*Allworth AE and Albertini DF (1993) Meiotic maturation in cultured bovine oocytes is accompanied by remodeling of the cumulus cell cytoskeleton Developmental Biology 158 101-112
Anderson E and Albertini DF (1976) Gap junctions between the oocyte and companion follicle cells in the mammalian ovary Journal of Cell Biology 71 680-686

*Antczak M and Van Blerkom J (1997) Oocyte influences on early development: the regulatory proteins leptin and STAT3 are polarized in mouse and human oocytes and differentially distributed within the cells of the preimplantation stage embryo Molecular Human Reproduction 3 1067-1086

*Buccione R, Schroeder AC and Eppig JJ (1990) Interactions between somatic cells and germ cells throughout mammalian oogenesis Biology of Reproduction 43 543-547

Can A and Albertini DF (1997) M-phase specific centrosome-microtubule alterations induced by the fungicide $\mathrm{MBC}$ in human granulosa cells Mutation Research 373 139-151

Can A, Holmes RM and Albertini DF (1997) Analysis of the mammalian ovary by confocal microscopy. In Microscopy of Reproduction and Development: A Dynamic Approach pp 101-108 Ed. PM Motta. Antonio Delfino Editore, Rome

Carabatsos MJ, Elvin J, Matzuk MM and Albertini DF (1998) Characterization of oocyte and follicle development in growth differentiation factor-9-deficient mice Developmental Biology 204 373-384

Dube JL, Wang P, Elvin J, Lyons KM, Celeste AJ and Matzuk MM (1998) The bone morphogenetic protein 15 gene is X-linked and expressed in oocytes Molecular Endocrinology 12 1809-1817

Elvin JA and Matzuk MM (1998) Mouse models of ovarian failure Reviews of Reproduction 3 183-195

Elvin JA, Clark AT, Wang P, Wolfman NM and Matzuk MM (1999a) 
Paracrine actions of growth differentiation factor-9 in the mammalian ovary Molecular Endocrinology 13 1035-1048

Elvin JA, Yan C, Wang P, Nishimori K and Matzuk MM (1999b) Molecular characterization of the follicle defects in the growth differentiation factor 9-deficient ovary Molecular Endocrinology 13 1018-1034

Elvin JA, Yan C and Matzuk MM (2000) Growth differentiation factor-9 stimulates progesterone synthesis in granulosa cells via a prostaglandin E2/EP2 receptor pathway Proceedings National Academy of Sciences USA $9710288-10293$

Galloway SM, McNatty KP, Cambridge LM et al. (2000) Mutations in an oocyte-derived growth factor gene (BMP15) cause increased ovulation rate and infertility in a dosage-sensitive manner Nature Genetics $\mathbf{2 5}$ 279-283

Grosse J, Bulling A, Brucker C, Berg U, Amsterdam A, Mayerhofer A and Gratzl M (2000) Synaptosome-associated protein of 25 kilodaltons in oocytes and steroid-producing cells of rat and human ovary: molecular analysis and regulation by gonadotropins Biology of Reproduction 63 643-650

Hayashi M, McGee EA, Min G, Klein C, Rose UM, van Duin M and Hsueh AJ (1999) Recombinant growth differentiation factor-9 (GDF-9) enhances growth and differentiation of cultured early ovarian follicles Endocrinology 140 1236-1244

Hertig AT and Adams EC (1967) Studies on the human oocyte and its follicle. I. Ultrastructural and histochemical observations on the primordial follicle stage Journal of Cell Biology 34 647-675

Kumar TR, Wang Y, Lu N and Matzuk MM (1997) Follicle stimulating hormone is required for ovarian follicle maturation but not male fertility Nature Genetics 15 201-204

Liu L, Dai Y and Moor RM (1997) Role of secreted proteins and gonadotrophins in promoting full maturation of porcine oocytes in vitro. Molecular Reproduction and Development 47 191-199

McGee EA and Hsueh AJ (2000) Initial and cyclic recruitment of ovarian follicles Endocrine Reviews 21 200-214
*McNatty KP, Heath DA, Lundy T, Fidler AE, Quirke L, O'Connell A, Smith P, Groome N and Tisdall DJ (1999) Control of early ovarian follicular development Journal of Reproduction and Fertility Supplement 54 3-16

Mermillod P, Oussaid B and Cognie Y (1999) Aspects of follicular and oocyte maturation that affect the developmental potential of embryos Journal of Reproduction and Fertility Supplement 54 449-460

* Motta PM, Makabe S, Naguro T and Correr S (1994) Oocyte follicle cells association during development of human ovarian follicle. A study by high resolution scanning and transmission electron microscopy Archives of Histology and Cytology 57 369-394

Nagyova E, Vanderhyden BC and Prochazka R (2000) Secretion of paracrine factors enabling expansion of cumulus cells is developmentally regulated in pig oocytes Biology of Reproduction 63 1149-1156

Rodgers RJ, van Wezel IL, Irving-Rodgers HF, Lavranos TC, Irvine CM and Krupa M (1999) Roles of extracellular matrix in follicular development Journal of Reproduction and Fertility Supplement 54 343-352

Rodgers RJ, Irving-Rodgers HF and van Wezel IL (2000) Extracellular matrix in ovarian follicles Molecular and Cellular Endocrinology 163 73-79

Solovyeva EV, Hayashi M, Margi K, Barkats C, Klein C, Amsterdam A, Hsueh AJ and Tsafriri A (2000) Growth differentiation factor-9 stimulates rat theca-interstitial cell androgen biosynthesis Biology of Reproduction 63 1214-1218

Suzuki H, Jeong BS and Yang X (2000) Dynamic changes of cumulus-oocyte cell communication during in vitro maturation of porcine oocytes Biology of Reproduction 63 723-729

Wassarman PM and Albertini DF (1994) The mammalian ovum. In The Physiology of Reproduction pp 79-122 Eds E Knobil and JD Neill. Raven Press, New York

Yoshida H, Takakura N, Kataoka H, Kunisada T, Okamura H and Nishikawa SI (1997) Stepwise requirement of c-kit tyrosine kinase in mouse ovarian follicle development Developmental Biology 184 $122-137$ 